\section{Resistance to Botryosphaeria dothidea in Pistachio}

\author{
D.E. Parfitt ${ }^{1}$ and N. Arjmand \\ Department of Pomology, One Shields Avenue, University of California, Davis \\ CA 95616
}

\section{T.J. Michailides}

Department of Plant Pathology, 9240 South Riverbend Avenue, Parlier, CA 93648

Additional index words. disease, panicle and shoot blight, inoculation, Fusicoccum sp.

\begin{abstract}
Procedures were developed to permit screening pistachio for resistance to infection by Botryosphaeria dothidia panicle and shoot blight. A method of growing $B$. dothidea in liquid culture was developed and two inoculation procedures, direct injection of inoculum into shoots at the base of leaf petioles and a leaf scratch assay, were used to test selected pistachio (Pistacia vera $\mathrm{L}$.) clones for resistance to $B$. dothidea. Both the direct injection and the scratch test procedures provided easily scored symptoms. Both solid and liquid cultures produced visible infections. Sources of resistance were identified in an F1 interspecific $P$. vera $\mathrm{cv}$. Sfax $\times$ P. integerrima hybrid cross and $\boldsymbol{P}$. integerrima genotypes. $P$. integerrima may be a valuable source of resistance for cultivar improvement, but resistant $P$. vera genotypes were not found. No correlation between Alternaria alternata resistance and $B$. dothidea resistance was found in $P$. vera.
\end{abstract}

Botryosphaeria dothidia (Moung.:Fr.) Ces. $\&$ De Not. is a marginally pathogenic fungus that is found on a variety of plants in various parts of the world as well as on California vegetation, including Sequoia L.. and Rubus L. Until 1998, it was not generally considered to be an economically important pathogen on pistachio, although Michailides (1991) warned that it could be a significant problem. Following several wet winters and late spring rains, a severe epiphytotic developed in Summer 1998 in the northern and central growing areas in California (from Glenn Co. to Madera Co.) Several years of conditions favorable for disease development, which permitted the development of a high level of inoculum in the field, followed by an unusually wet summer, caused widespread crop loss due to panicle infection and death. The specific conditions needed for disease development have been described (Ma et al., 2001; Michailides,1991; Michailides and Morgan, 1992; Ntahimpera et al., 2002), but the conditions needed for a field epiphytotic are not known. Arecent study using molecular markers by Smith et al. (2001) has suggested that the $B$. dothidia isolates collected from pistachio are actually representatives of a Fusicoccum sp. Ma and Michailides (2002), using similar markers, found that the pistachio isolates were very different from previously described $B$. dothidia isolates but did not classify them as members of a separate genus.

Pistacia vera L. cv. Kerman, the primary California cultivar, has been shown to be quite

Received for publication 17 June 2002. Accepted for publication 30 Sept. 2002. We wish to thank H.M. Chan for assistance culturing $B$. dothidea samples. This research was funded in part by the California Pistachio Commission.

${ }^{1}$ To whom reprint requests should be sent. E-mail address: deparfitt@ucdavis.edu vulnerable to infection by the asexual spores of $B$. dothidea if appropriate environmental conditions are present. Resistant cultivars or sources of resistance for cultivar development are needed. Recommended pruning and fungicide spray control measures can cost growers $\$ 400$ to $\$ 800 /$ acre to implement. An alternative approach is to breed new resistant cultivars. The following report describes the development of protocols producing reproducible symptoms, and identification of pistachio germplasm with resistance to $B$. dothidea infection.

Environmental conditions were favorable for development of Botryosphaeria blight in 1998 and initial examination of three fruiting female full-sib progeny from a $P$. vera $\mathrm{x}$ $P$. integerrima Stewart cross suggested that the cross was segregating for resistance. One tree appeared to be highly susceptible with most clusters and nuts dead (>80\% damaged panicles), while the other two trees had completely unaffected panicles and nuts. To explore the possibility that sources of genetic resistance are present in pistachio, a procedure to produce reproducible infection in nonfruiting tissue was needed. A fruit-based assay was considered to be less useful because fruit are only produced once a year and the specific conditions needed for a successful infection process could not be easily manipulated on a single tree basis. Toothpick inoculation is a traditional approach to fungal inoculation in annual field crops (Hildebrand, 1952; Pryor et al., 2000). This method could not be used for testing the pistachio materials because of the woody nature of the tree branches and our desire to expose the tree to a large quantity of the weakly pathogenic fungus. Therefore a modified procedure using mass infusion of liquid inoculum was used. A procedure based on our prior experience with a leaf scratch test for Alternaria alternata (Fries) Keissler was also evaluated (Aradhya et al., 2001).

\section{Materials and Methods}

Plant materials. A small progeny family from a $P$. vera $\mathrm{cv}$. Sfax $\times$ P. integerrima cross was planted at the Univ. of California Wolfskill Experimental Orchard (WEO) in 1990 as part of a larger plant breeding program. Trees 65 , 71,75 , and 77 from the cross were selected for the injection experiment in 1999, as well as for a leaf scratch assay used previously for testing for A. alternata (Aradhya et al., 2001). Tree 79 at WEO from a $P$. integerrima $\times P$. integerrima cross was also tested.

Ten seedling $P$. vera $\times P$. vera progeny and one $P$. integerrima tree at the Kearney Agricultural Center (KAC), were tested to determine whether there was a correlation between A. alternata resistance and B. dothidea resistance. Alternaria alternata scores from Chao et al. (2001) were correlated with data from this study. Two 'Kerman' trees (known to be susceptible) at WEO were tested in Sept. 2000 with the direct injection and leaf scratch protocols, to test the viability of liquid cultured inoculum.

Construction of inoculum: solid medium culture. Stem lesions were collected from WEO in 1999. Pycnidia were isolated and cultured on potato dextrose agar (PDA). Single hyphal tip subcultures were taken from the resulting mycelial mat and were grown in PDA plates. Dr. Michailides verified that the resulting cultures were $B$. dothidea. A $1-\mathrm{cm}^{2}$ section of the fungal mat consisting of both mycelial and conidial fragments was cut from the main mass of growth and suspended in sterile distilled water at a total volume of 10 $\mathrm{mL}$ under sterile conditions $(50 \%$ by volume suspension). This suspension was vortexed so that no fungal fragments $>0.5 \mathrm{~mm}$ were visible.

Construction of inoculum: liquid medium culture. Inoculations in 2000 were performed from an initial liquid culture system. Acidified potato dextrose broth (APDB) was found to be the most effective medium for producing large scale in-vitro cultures of $B$. dothidea. PDA was prepared as per Bacto-Agar (Fisher Scientific, Pittsburgh) manual recommendations: [(B13), $200 \mathrm{~g}$ Dehydrated Potato, infusion, $20 \mathrm{~g}$ Bacto-Dextrose, 156 Bacto-Agar]. The APDB was prepared by addition of 2.6 $\mathrm{mL}$ of a $10 \%$ lactic acid solution under sterile conditions to $1000 \mathrm{~mL}$ of warm $\left(55^{\circ} \mathrm{C} 0.8 \%\right.$ PDA solution ( $8 \mathrm{~g}$ of PDA powder per liter of distilled water autoclaved at $20 \mathrm{psi}$ and $121^{\circ} \mathrm{C}$ for $20 \mathrm{~min}$ ). The APDB was cooled to $25^{\circ} \mathrm{C}$ and a small amount of $B$. dothidea source culture, scraped from growing hyphal tips, was added to the broth culture. The inoculated broth was gently shaken at 25 to $28^{\circ} \mathrm{C}$ under low light in 1- or 2-L Erlenmeyer flasks. After 14 to $21 \mathrm{~d}$, a large number of creamy white globular masses of fungal mycelia, $\approx 1 \mathrm{~cm}$ in diameter, formed in the broth solution. When the masses of fungal mycelia occupied $90 \%$ of the volume of the flasks, they were transferred to 2- or 5-L Erlenmeyer flasks at $4{ }^{\circ} \mathrm{C}$ without additional 
shaking. This procedure promoted the formation of a mycelial mat on the surface of the suspension, and the growth and differentiation of conidia which gave the surface of the fungal mat a dirty gray-black fluffy texture within a week after transfer to $4{ }^{\circ} \mathrm{C}$.

The inoculum consisted of a mixture of hyphae and conidia. Identity of the inoculum was verified by T.J. Michailides, both on solid media and in liquid culture on two separate occasions.

Directinoculation by injection of individual trees. Direct injection of the fungus into the vascular system of the tree was thought to be the most reliable approach for induction of infection. Direct inoculations were done in 1999 at WEO and KAC with an inoculum suspension derived from the solid medium culture. Three treatments and three water controls were administered to trees $65,71,75,77$, and 79 at WEO. Three treatment inoculations and three water controls were done per replicate at KAC. The score was the average of the observed infection level $(0=$ no symptoms, $3=$ some leaflets dead, lesions at site of injection, $5=$ petioles, leaves, and leaflets dead, spreading lesions). Observations were taken after three weeks. Trees were considered resistant if scored as zero and susceptible if scored greater than two. Inoculations in 2000 used a suspension derived from the liquid culture medium. Three direct injections of inoculum and water controls were applied to the first tree, while four injections and four controls were applied to the second tree.

A 0.2-cc volume of inoculum or sterile water control was injected into the cambium at the at the base of leaf petioles on actively growing terminal shoots. Injection sites were wrapped with parafilm to prevent the injection site from drying and secondary infection by opportunistic pathogens (Fig. 1A). A 1.0-cc capacity sterile disposable syringe (TB type), and an 18 gage, $1.9 \mathrm{~cm}$ long, sterile winged infusion hypodermic needle was used to inject the inoculum. Infected and control shoots were collected and sectioned lengthwise for observation of stem lesions.

Leaf scratch assay. A sterilized scalpel was used to break the upper cuticular layer of the leaflet (a scratch) and thus create an entry point for the fungus. Sterile filter paper soaked with $1 \mathrm{ml}$ of inoculum or sterile distilled water as control was applied to the leaflet surface after scratching. The filter paper was attached to the leaflet surface using masking tape and the leaflet was wrapped with cellophane to prevent desiccation. A positive result was indicated by the appearance of spreading lesions on the leaf and eventual leaf and petiole necrosis. The leaf scratch assay was tested on the segregating $P$. vera $\times$ P. integerrima progeny previously used for the direct injection protocol in 1999. In 2000 , the assay was tested on two 'Kerman' trees at WEO with three or four water controls and three or four treatments per tree, respectively.

Data analysis. Spearman and Pearson correlations were performed on the data collected from the $P$. vera trees at KAC with SAS ver. 8.2 (SAS Institute, 2002). The arcsin transformation (Steel and Torrie, 1960) was applied to the data prior to computation of the Pearson correlation. A general linear models analysis of variance was performed with SAS ver. 8.2 on the transformed Botryosphaeria scores, including least squares means, F test, significance level, and least significant difference (LSD). Type III sums of squares were used to adjust for the missing data points. Least squares means and LSDS were back transformed for presentation in Table 1. Yates correction was used for $\mathrm{X}^{2} \mathrm{~s}$.

\section{Results}

Inoculation by injection of individual trees. The procedure produced clearly scorable symptoms in trees 71, 75, and 77 from the $P$. vera $\mathrm{x}$ P. integerrima hybrid (Fig. 1B) while the water controls produced no symptoms (Fig.
1C). Leaflet wilting was observed within 1 week, and by 3 weeks, some or all of the leaves on the infected shoots (depending on severity of infection) had turned brown and dried. Tree 65, which had shown a high level of panicle damage in 1998, also had large 4 to 5-cm lesions at infection points while tree 71, which showed little panicle damage in 1998, had no leaf symptoms and only small $1-\mathrm{cm}$ stem lesions. By the end of the growing season the stem lesions had expanded to $\approx 2 \mathrm{~cm}$ (Fig. 1D), but were still less than one half the size of the lesions in tree 65 (Fig. 1E). Trees 75 and 77 , which are males and thus did not have observable panicle damage in 1998, showed susceptible reactions after inoculation (wilted leaves, spreading stem lesions). Tree 79, from a $P$. integerrima $\times$ P. integerrima cross and the the $P$. integerrima tested at KAC, developed no lesions or other symptoms.

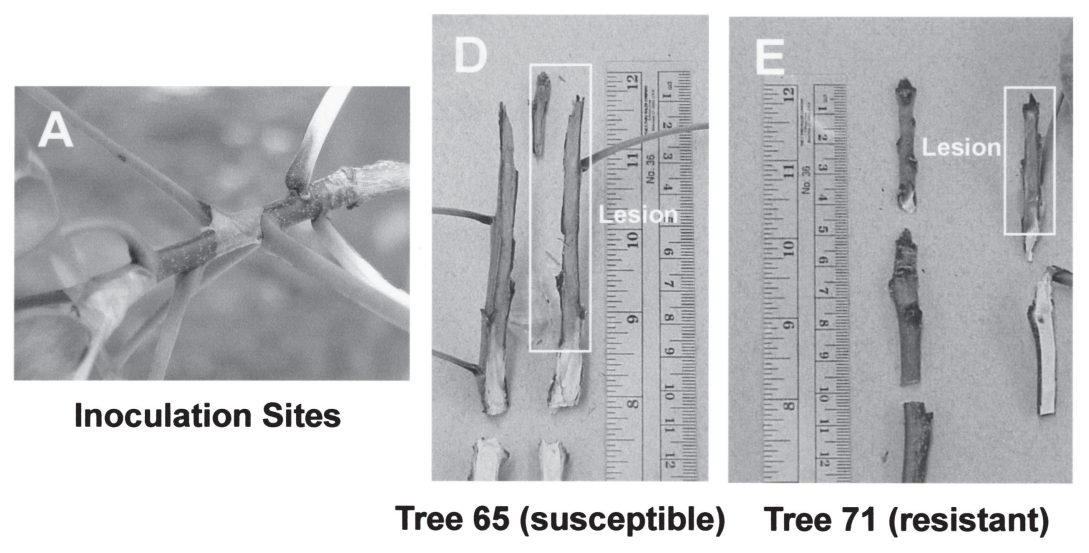

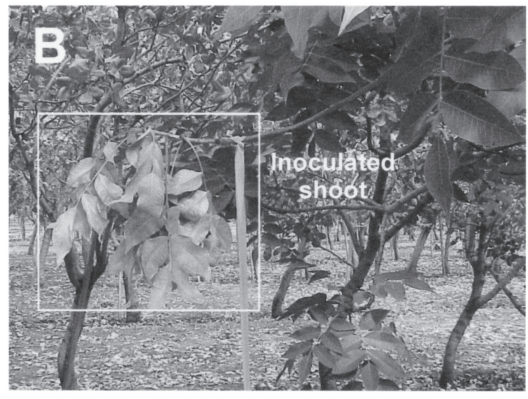

Tree 65 (susceptible)

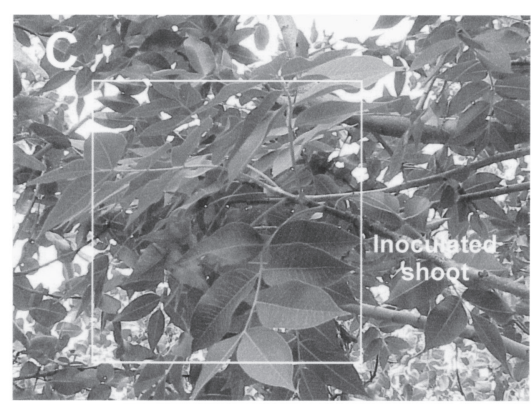

Tree 71 (resistant)

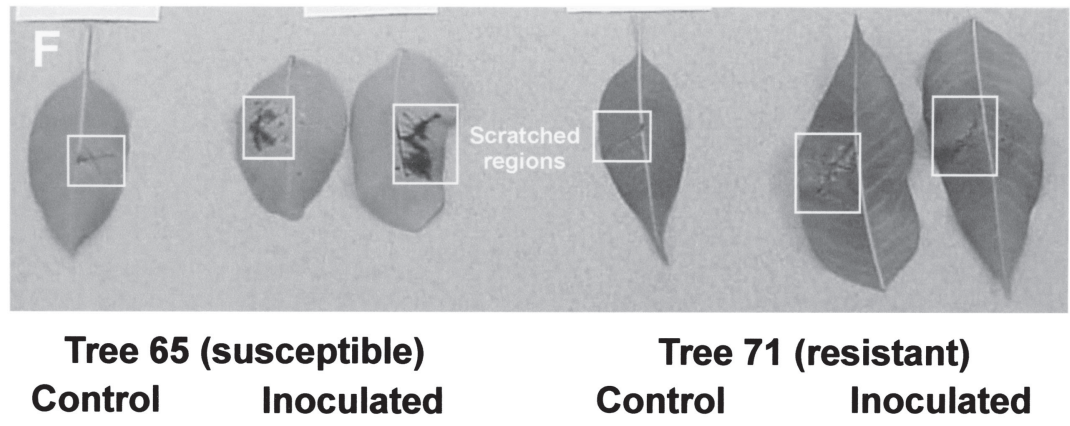

Fig. 1. Shoot showing sites injected with $B$. dothidea (A). Leaf symptoms from direct inoculation (injection) of $B$. dothidea inoculum into leaf petiole base; tree 65 susceptible (B), tree 71 resistant (C). Cross section of stems of resistant and susceptible siblings, showing differential progression of infection; tree 65 susceptible (D), and tree 71 resistant (E). Leaf scratch test inoculation for susceptible (tree 65) and resistant (tree 71) pistachio using water control and B. dothidea inoculum, $7 \mathrm{~d}$ after inoculation (F). 
Table 1. Test of Alternaria alternata resistant genotypes at Kearney Agricultural Center for resistance to $B$. dothidea in 1999 .

\begin{tabular}{|c|c|c|c|c|c|c|c|}
\hline Cross & Clone no. & $\begin{array}{l}\text { Alternaria } \\
\text { resistance }\end{array}$ & $\begin{array}{c}\text { Replicate }^{\mathrm{z}} \\
1\end{array}$ & $\begin{array}{c}\text { Replicate } \\
2\end{array}$ & $\begin{array}{c}\text { Least } \\
\text { square means }\end{array}$ & Control & Status' \\
\hline P.vera & a2-42 & 1 & 4.5 & 0.0 & 1.7 & 0 & $\mathrm{~m}$ \\
\hline P. vera & a14-80 & 1 & 0.5 & no data & 0.5 & 0 & $\mathrm{~m}$ \\
\hline P. vera & a22-80 & 1 & 0.5 & 3.0 & 1.6 & 0 & $\mathrm{~m}$ \\
\hline P. vera & a5-79 & 2 & 5.0 & 5.0 & 5.0 & 0 & $\mathrm{~s}$ \\
\hline P. vera & a19-80 & 2 & 0.5 & 2.0 & 1.7 & 0 & $\mathrm{~m}$ \\
\hline P. vera & a8-46 & 3 & 0.5 & no data & 0.5 & 0 & $\mathrm{~m}$ \\
\hline P. vera & a9-62 & 3 & $1.5^{\mathrm{x}}$ & 0.0 & 0.4 & 0 & $\mathrm{r}$ \\
\hline P. vera & a21-80 & 3 & 2.0 & 3.0 & 2.5 & 0 & $\mathrm{~s}$ \\
\hline P. vera & b21-78 & 3 & 0.0 & 0.0 & 0.0 & 0 & $\mathrm{r}$ \\
\hline P. vera & b22-77 & 3 & 0.5 & 0.5 & 0.5 & 0 & $\mathrm{~m}$ \\
\hline P. integerrima & b9-30 & 2 & 0.0 & 0.0 & 0.0 & 0 & $\mathrm{r}$ \\
\hline \multicolumn{8}{|c|}{$A N O V A^{w}$} \\
\hline $\mathrm{F}$ & & & & & 2.36 & & \\
\hline Prob. $>$ F & & & & & 0.12 & & \\
\hline $\mathrm{LSD}^{\mathrm{v}}$ & & & & & 0.9 & & \\
\hline
\end{tabular}

${ }^{0} 0=$ no symptoms, 3 = some leaflets dead, lesions at site of injection, $5=$ petioles, leaves, and leaflets dead, spreading lesions.

${ }^{y_{S}}=$ susceptible, $\mathrm{m}=$ somewhat susceptible, and $\mathrm{r}=$ resistant.

${ }^{x}$ Damage was due to Alternaria alternata infection.

"Analysis of variance (ANOVA) performed on P. vera, only.

'Least significant difference (LSD) at 5\% significance level.

Although some nonsignificant differences in resistance during the first one to three weeks after infection were observed among the $P$. vera progeny and some initially appeared to be resistant (Table 1), they all eventually showed symptoms of $B$. dothidea infection. A slightly negative, but nonsignificant correlation between $A$. alternata and $B$. dothidea resistance was observed $($ Pearson $r=-0.3$, Prob $>|r|=0.4$; Spearman $r=-0.3$, Prob $>|r|=0.3)$. Therefore, the hypothesis of a general fungus resistance mechanism in $P$. vera was rejected.

The $B$. dothidea inoculum cultured in liquid medium, and tested on 'Kerman' in 2000, produced symptoms in 5 of 7 (dried leaflets, petiole necrosis, score of three or more) tests, while 6 of 7 control treatments produced no symptoms $\left(\mathrm{X}^{2}=0.36, \mathrm{P}=0.55\right)$. 'Kerman' was shown to be susceptible, a result that was also clearly demonstrated by the epiphytotic of 1998, which caused significant crop loss to the pistachio industry.

Leaf scratch assay. The leaf scratch test protocol produced symptoms on the most susceptible tree ( $P$. hybrid tree 65$)$ while water controls showed no symptoms (Fig. 1F). Tree 71, previously identified as moderately resistant, showed only minor symptom development. In 'Kerman' trees tested in 2000, all four of the inoculated leaves developed symptoms after three weeks and 2 of 4 control leaves also showed some necrosis $\left(\mathrm{X}^{2}=0.63, P=0.32\right)$. However, leaf damage to the controls was qualitatively different from the $B$. dothidea treatments. B. dothidea treated leaflets showed clear evidence of fungal development, black discoloration and sporulation, while the control treatment leaflets had light brown areas as the result of physical damage to the leaf.

\section{Discussion}

Our direct injection protocol delivers the inoculum into the cambial area and increases the probability of infection because the fungus is delivered directly to the most susceptible tissues. Loss of fungus viability due to dessication is avoided. A major benefit of the injection protocol is the ability to score male trees or those that have not yet flowered. Our leaf scratch assay which was tested as an alternative for the direct injection procedure showed promising results during initial tests. However, while application of inoculum was easier with the leaf scratch assay, symptom expression was much stronger with the direct inoculation protocol. Inoculum dessication (and associated loss of inoculum viability) was more of an issue with the leaf scratch procedure. Identification of infected vs. noninfected leaf tissues was also more difficult because necrosis occurred on some of the noninfected tissues due to mechanical damage. However, both procedures produced symptoms that could be easily distinguished from controls. The test in 2000 also showed that cultured liquid inoculum could be used to induce symptomatic infections.

Several Pistacia hybrid genotypes were identified that showed significant resistance to $B$. dothidea using the direct inoculation and/or leaf scratch protocols. The results of controlled vegetative bud/petiole and leaf scratch inoculations of $P$. vera, $P$. integerrima x. P. vera hybrids, and $P$. integerrima with $B$. dothidea mycelial and conidial fragments at WEO and KAC conducted in Summer and early Fall 1999, suggest that $P$. integerrima is a source for resistance to $B$. dothidea.
Because $P$. integerrima does not produce an economically acceptable nut, a backcrossing program to incorporate resistance genes into acceptable $P$. vera cultivars would be needed to develop resistant cultivars. However, with the continuing development of molecular genetic technologies, resistance genes may be directly isolated and incorporated into commercial $P$. vera cultivars.

Some differences were observed in the level of susceptibility among the $P$. vera genotypes that were tested, but none were observed to be completely resistant to infection. Informal observations of the P. vera seedling population at WEO during and after the 1998 epiphytotic suggest that some differences in resistance may be present in $P$. vera. However, to obtain high levels of resistance, incorporation of genes from $P$. integerrima will be necessary.

\section{Literature Cited}

Aradhya, M.K., H.M. Chan, and D.E. Parfitt. 2001. Genetic variability in the pistachio late blight fungus, Alternaria alternata. Mycological Res. 105:300-306.

Chao, C.T., D.E. Parfitt, and T.J. Michailides. 2001. Alternaria late blight (Alternaria alternata) resistance in pistachio (Pistacia vera) and selection of resistant genotypes. J. Amer. Soc. Hort. Sci. 126:481-485.

Hildebrand, A.A. 1952. An elaboration of the toothpick method of inoculating plants. Can. J. Agr. Sci.33:506-507.

Ma, Z. and T.J. Michailides. 2002. Characterization of Botryosphaeria dothidea isolates collected from pistachio and other plant host in California. Phytopathology 92:519-526.

Ma, Z., D.P. Morgan, and T.J. Michailides. 2001. Effects of water stress on Botryosphaeria blight of pistachio caused by Botryosphaeria dothidea. Plant Dis. 85:745-749.

Michailides, T.J. 1991 Pathogenicity, distribution, sources of inoculum, and infection courts of Botryosphaeria dothidea on pistachio. Phytopathology 81:566-573

Michailides, T.J. and D.P. Morgan. 1992. Effects of temperature and wetness duration of infection of pistachio by Botryosphaeria dothidea and management of disease by reducing duration on irrigation. Phytopathology 82:1399-1406.

Ntahimpera, N., G.F. Driever, D. Felts, D.P. Morgan, and T.J. Michailides. 2002. Dynamics and pattern of latent infection caused by Botryosphaeria dothidea on pistachio buds. Plant Dis. 86:282-287.

Pryor, B.M., R.M. Davis, and R.L. Gilbertson. 2000 A toothpick inoculation method for evaluating carrot cultivars for resistance to Alternaria radicina. HortSci. 35:1099-1102.

SAS Institute. 2002. SAS ver. 8.2. SAS Inst. Cary, N.C.

Smith, D.R., T.J. Michailides, and G.R. Stanosz. 2001. Differentiation of a Fusicoccum $\mathrm{sp}$. causing panicle and shoot blight on California pistachio trees from Botryosphaeria dothidea. Plant Dis. 85:1235-1240.

Steel, R.G.D. and J.H. Torrie. 1960. Principles and procedures of statistics. McGraw-Hill. New York. $481 \mathrm{p}$. 